\title{
Analysis of Financial Performance of Commercial Banks Listed in Indonesia Stock Exchange
}

\author{
Jojor Lisbet Sibarani \\ Politeknik Negeri Medan \\ Cahyo Ginarti \\ Politeknik Negeri Medan \\ Meily Surianti \\ Politeknik Negeri Medan
}

\begin{abstract}
The purpose of this study is to analyze the financial performance of commercial banks listed on the Indonesia Stock Exchange. For the short term, the results of this study are expected to determine the financial performance of commercial banks listed on the Indonesia Stock Exchange during the observation period. Financial performance is one of the most important elements on which investors base their decisions. The results of this study are not only useful for investors, but also useful for the general public who want to use banking services, in terms of choosing a healthy bank to save the funds they have. For the long term, the results of this study are expected to contribute to the field of accounting, especially financial accounting courses and financial statement analysis. The results of this study can be used as material for compiling case studies in both courses and used in the learning process. The research objective can be achieved by collecting secondary data in the form of a company's financial statements that are the subject of research, tabulated data, and then analyzed using the time series method and trend analysis.
\end{abstract}

Keywords: Banking, Ratio, Financial Performance

DOI: $10.7176 / \mathrm{EJBM} / 12-3-17$

Publication date: January $31^{\text {st }} 2020$

\section{Background of Study}

In modern society, employees must have analytical, systematic and unusual thinking. In most countries, banks are regulated by national governments or central banks. Commercial banks usually focus on managing withdrawals and receiving deposits and providing short-term loans to individuals and small businesses. Commercial banks usually focus on managing withdrawals and receiving deposits and providing short-term loans to individuals and small businesses. Consumers mainly use these banks for checking accounts and basic savings, certificates of deposit, and home mortgages.

Although a country's economic development depends on a number of factors such as industrial growth and development, agricultural modernization, expansion of domestic and foreign trade, political stability, its dependence on the largest level in the banking sector is undeniable and / or banks play a key role in increasing economic efficiency by channeling funds from surplus unit resources to those who have limited access and / or who need it. Misra and Asphalt (2013).

Examining the existing phenomena, research aimed at identifying the financial performance of banks in Indonesia is felt very necessary. This study will identify and analyze the financial performance of commercial banks listed on the Indonesia Stock Exchange, especially to see the impact of the weakening of the rupiah against the financial performance of banks in Indonesia. The selection of commercial banks listed on the Indonesia Stock Exchange as a research subject is given that banks that have been listed on the stock exchange have a very big influence on market valuations, because as companies that have gone public, the financial performance of these companies will always be highlighted by the decision makers in the market. 


\section{Literature Review}

\section{Analysis of Financial Performance and Decision Making}

Financial analysis is a structural and logical way to present the overall financial performance of a financial institution. It also helps to evaluate and make decisions for business operations. In the financial analysis process, ratio analysis is the most dominant and logical structure to help stakeholders related to business. In the process of financial ratio analysis there are several categories for the same field of financial institutions. So business stakeholders try to concentrate on getting an overall business review of profitability, liquidity, asset management and solvency ratio analysis. These ratios not only help the decision making process but also emphasize risk avoidance and factors related to increased profits. To calculate this ratio, it is necessary to take quantitative data from the trading activities of banks and other sources. (Islam, 2014).

Analysis of financial performance is very important for a company to be able to compete in a very competitive market as it is today. Financial performance analysis is an assessment of the feasibility, solidity and soundness of a business, sub-business or mission. Gepp and Kumar (2008) incorporate the "bias" factor of time into the classical business failure prediction model. Using Altman (1968) and Ohlson (1980) models for matching samples from failed and non-failing companies from 1980, they found that the accuracy of the Altman model predictions decreased when applied to 1980 data. These findings explain the importance of including the time factor in the prediction model traditional failure.

\section{Measurement of Financial Performance at the Bank}

Various ratios that have been used in research to assess bank financial performance include Net Interest Margin (NIM), Return on Assets (ROA), and Return on Equity by Ahmed (2003), profitability ratios according to Akinola (2008) including Profit before Tax (PBT), Profit after Tax (PAT), ROE, and Return on Capital (ROC). Some other studies on profitability also use average bank asset return (ROAA), net interest margin (NIM) and average return on equity (ROAE) to measure profitability. Francis (2013). However, because of differing views among scholars about the superiority of one indicator over another as a measure of profitability, there is no clear boundary as to which is the most suitable. (Tekatel, 2019). Nevertheless, most of the literature limits the size of profitability to only three widely used measures namely Return on Assets (ROA), Return on Equity (ROE), and Net Interest Margin (NIM).

\section{Ratios for Measurement of Bank Financial Performance}

\section{Liquidity}

Liquidity shows the ability of banks to meet their financial obligations in a timely and effective manner. Samad (2004) states that 'liquidity is the life and blood of commercial banks'. Financial liabilities are withdrawn through retail and wholesale distribution channels. The resulting retail funding is considered to be less elastic and more reliable than deposits withdrawn from wholesale distribution channels (Thygerson, 1995). The following ratio is used to measure liquidity.

1. Net Loans to total asset ratio $($ NLTA $)=$ Net loans $/$ total assets.

NLTA measures the percentage of assets related to loans. The ratio of net loans to total assets (NLTA) is also an important ratio that measures the condition of bank liquidity. Whereas Loan to Deposit is the ratio in which bank liquidity is measured in terms of deposits, NLTA measures bank liquidity in terms of total assets.

\section{Loans to deposit Ratio $(\mathrm{LDR})=$ Loans/total deposits.}

Loan to deposit is the most important ratio to measure the condition of bank liquidity. Here, a loan means a down payment for a conventional bank. Banks with low LDR are considered to have excessive liquidity, lower profit potential, and therefore less risk compared to banks with high LDR.

\section{Profitability Ratio}

The profitability ratio helps measure how well the company manages its expenses. This measurement allows evaluating the company's profit with respect to a certain level of sales, a certain level of assets, or owner's investment. This study uses Return on Assets $(\mathrm{ROA})=$ net profit / total assets showing the ability of management to obtain deposits at reasonable costs and invest them in profitable investments (Ahmed, 2009). In general, a higher ratio means better managerial performance and efficient utilization of company assets and a lower ratio is an 
indicator of inefficient use of assets.

The use of ROA as a profitability measure for this particular study is due to the limitations of Net Interest Margin (NIM) and Return on Equity (ROE). NIM is reported to have two main limitations. First, it does not measure the bank's total profitability because most of them get fees and other non-interest income through services such as brokers and deposit account services without taking into account operating costs, such as personnel and facility costs, or credit fees. In addition, the net interest margins of the two banks cannot be contrasted because the two banks are separated in their own way in the nature of their activities, the composition of the customer base, etc. (Tekatel, 2019)

\section{Credit Performance}

Credit risk is one of the main risks faced by banks. This can be described as a potential loss arising from the failure of the counterparty to carry out in accordance with contractual arrangements with the bank. Failures may arise due to the unwillingness of the opposing party or deterioration in economic conditions etc. (Islam, 2014). Bank risk management has been designed to overcome all of these problems. While it is expected that banks will bear some bad loans and losses in their loan activities, one of the main objectives of the bank is to minimize these losses (Casu et al, 2006).

To measure credit performance, the following ratios are used: Nonperforming Loans to Total Loans (NPLTL) = Nonperforming Loans / Total loans. This ratio shows the proportion of total loans that have been set aside but not charged. This is the percentage of the total loans that have defaulted or near defaulted. After a loan has performed poorly, the chance that it will be repaid in full is considered far lower.

\section{Research Methods}

This research is a quantitative study, which calculates performance ratios in the form of liquidity ratios, profitability and credit performance. The performance ratio will be analyzed using trend analysis and time series analysis.

The data collected is secondary data, which is derived from the financial statements of commercial banks which are the research samples in 2013 - 2018. There are 44 (forty four) commercial banks listed on the Jakarta Stock Exchange. Samples were selected using purposive sampling. Financial reports can be accessed on the Indonesia Stock Exchange website. Data is processed to obtain the ratio of financial performance during the observation year using Excel and SPSS. Analysis of the data used is trend analysis and time series analysis. Trend Analysis is looking at the development of the three ratios which are the research parameters, namely liquidity ratios, profitability ratios and credit performance ratios from banks which are the research samples during the observation period. Whereas time series analysis is looking at comparison of ratios that are parameters from year to year.

\section{Research Results}

Return on Asset is shown in Table 1.

This ratio shows the rate of return (profit) compared to total assets. The higher this ratio shows the better performance. The summary of data processing results shows that the most decline in ROA occurred in 2017, namely 23 banks or $62.16 \%$ of the total banks studied. Interestingly, in 2018 the decline in ROA occurred only in 17 banks or $45.95 \%$ of the total banks studied, lower than in 2015 which reached 20 banks or $54.05 \%$ and in 2017 which became 23 banks or $62.16 \%$ of the total banks studied. 
Table 1. Return on Asset

\begin{tabular}{|c|c|c|c|c|c|}
\hline $\mathrm{NO}$ & KETERANGAN & 2015 & 2016 & 2017 & 2018 \\
\hline 1 & BSWD (BANK OF INDIA INDONESIA) & MENURUN & MENURUN & MENINGKAT & MENINGKAT \\
\hline 2 & AGRO (PT BANK RAKYAT INDONESIA AGRONIAGA TbK) & MENURUN & MENINGKAT & MENINGKAT & MENURUN \\
\hline 3 & AGRS (PT BANK AGRIS Tbk) & MENURUN & MENURUN & MENURUN & MENURUN \\
\hline 4 & BABP (PT BANK MNC INTERNASIONAL Tbk) & MENINGKAT & MENURUN & MENURUN & MENINGKAT \\
\hline 5 & BACA (PT BANK CAPITAL INDONESIA Tbk) & MENURUN & MENINGKAT & MENURUN & MENURUN \\
\hline 6 & BANK DANAMON & MENURUN & MENINGKAT & MENURUN & MENINGKAT \\
\hline 7 & BANK TJURST & MENURUN & MENINGKAT & MENINGKAT & MENURUN \\
\hline 8 & BBKP (BANK BUKOPIN) & MENINGKAT & MENINGKAT & MENURUN & MENURUN \\
\hline 9 & BBMD (PT BANK MESTIKA DHARMA TbK) & MENURUN & MENINGKAT & MENURUN & MENURUN \\
\hline 10 & BBNI (BNI) & MENINGKAT & MENURUN & MENINGKAT & MENURUN \\
\hline 11 & BBRI (BRI) & MENURUN & MENINGKAT & MENURUN & MENURUN \\
\hline 12 & BBTN ( PT BANK TABUNGAN NEGARA Tbk) & MENINGKAT & MENINGKAT & MENURUN & MENURUN \\
\hline 13 & BEKS (BANK PEMBANGUNAN DAERAH BANTEN) & MENURUN & MENURUN & MENINGKAT & TETAP \\
\hline 14 & BINA (BANK INA PERDANA) & MENINGKAT & MENURUN & MENINGKAT & MENURUN \\
\hline 15 & BJBR (BANK PEMBANGUNAN DAERAH JAWA BARAT) & MENURUN & MENURUN & MENINGKAT & MENINGKAT \\
\hline 16 & BJTM (BANK DAERAH JAWA TIMUR) & MENURUN & MENINGKAT & MENINGKAT & MENURUN \\
\hline 17 & BKSW (BANK QNB INDONESIA) & MENINGKAT & MENURUN & MENURUN & MENINGKAT \\
\hline 18 & BMAS (BANK MASPION INDONESIA Tbk PT) & MENINGKAT & MENURUN & MENURUN & MENINGKAT \\
\hline 19 & BMRI (BANK MANDIRI PERSERO Tbk PT) & MENURUN & MENINGKAT & MENURUN & MENURUN \\
\hline 20 & BNBA (BANK BUMI ARTA) & MENURUN & MENINGKAT & MENINGKAT & MENINGKAT \\
\hline 21 & BNGA (BANK CIMB NIAGA) & MENURUN & MENINGKAT & MENURUN & MENINGKAT \\
\hline 22 & BNII (BANK MAYBANK INDONESIA TbK PT) & MENINGKAT & MENINGKAT & MENURUN & MENINGKAT \\
\hline 23 & BNLI (BANK PERMATA) & MENINGKAT & MENURUN & MENINGKAT & MENURUN \\
\hline 24 & BRIS (Bank BRIsyariah Tbk) & MENINGKAT & MENINGKAT & MENURUN & MENURUN \\
\hline 25 & BSIM (BANK SINARMAS) & MENINGKAT & MENURUN & MENURUN & MENURUN \\
\hline 26 & BTPN (Bank BTPN) & MENURUN & MENINGKAT & MENURUN & MENINGKAT \\
\hline 27 & BVIC (Bank Victoria International) & MENINGKAT & MENURUN & MENINGKAT & MENINGKAT \\
\hline 28 & DNAR (BANK DINAR INDONESIA Tbk) & MENURUN & MENURUN & MENURUN & MENINGKAT \\
\hline 29 & INPC (BANK ARTHA GRAHA INTERNASIONAL TbK PT) & MENURUN & MENINGKAT & MENURUN & TETAP \\
\hline 30 & MAYA (BANK MAYAPADA INTERNASIONAL TbK) & MENINGKAT & MENINGKAT & MENURUN & MENURUN \\
\hline 31 & MCOR ( BANK CHINA CONSTRACTION INDONESIA ) & MENINGKAT & MENURUN & MENINGKAT & MENINGKAT \\
\hline 32 & MEGA ( BANK MEGA ) & MENINGKAT & MENINGKAT & MENURUN & MENURUN \\
\hline 33 & NISP( BANK OCBC) & MENURUN & MENINGKAT & MENINGKAT & MENINGKAT \\
\hline 34 & NOBU ( BANK NATIONAL NOBU) & TETAP & MENINGKAT & MENURUN & MENINGKAT \\
\hline 35 & PNBN ( BANK PAN INDONESIA) & MENURUN & MENINGKAT & MENURUN & MENINGKAT \\
\hline 36 & PNBS ( BANK PANIN SYARIAH) & MENURUN & MENURUN & MENURUN & MENINGKAT \\
\hline 37 & SDRA ( BANK WOORI SAUDARA ) & MENINGKAT & MENINGKAT & MENINGKAT & MENINGKAT \\
\hline & KINERJA KEUANGAN MENINGKAT & 16 & 22 & 14 & 18 \\
\hline & KINERJA KEUANGAN MENURUN & 20 & 15 & 23 & 17 \\
\hline & KINERJA KEUANGAN TETAP & 1 & 0 & 0 & 2 \\
\hline & TOTAL & 37 & 37 & 37 & 37 \\
\hline & \% ROA MENINGKAT & $43.24 \%$ & $59.46 \%$ & $37.84 \%$ & $48.65 \%$ \\
\hline & $\%$ ROA MENURUN & $54.05 \%$ & $40.54 \%$ & $62.16 \%$ & $45.95 \%$ \\
\hline & $\%$ ROA TETAP & $2.70 \%$ & $0.00 \%$ & $0.00 \%$ & $5.41 \%$ \\
\hline & $\%$ TOTAL & $100 \%$ & $100 \%$ & $100 \%$ & $100 \%$ \\
\hline
\end{tabular}

\section{ROA}

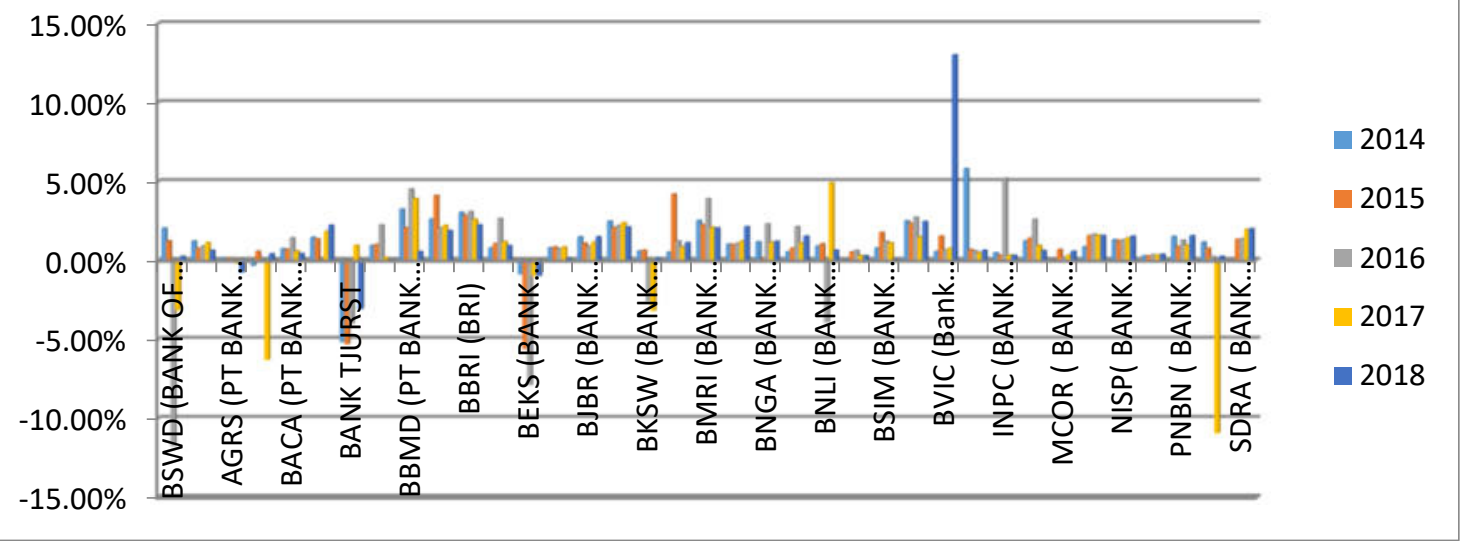

Figure 1. Return on Asset 
From the bar chart above, it can be seen that BIVC or Bank Victoria International experienced a very high ROA surge compared to other banks in 2014 and 2018.

\section{Loan to Deposit Ratio}

Table 2. Loan to Deposit Ratio

\begin{tabular}{|c|c|c|c|c|c|}
\hline NO & KETERANGAN & 2015 & 2016 & 2017 & 2018 \\
\hline 1 & BSWD (BANK OF INDIA INDONESIA) & MENURUN & MENURUN & MENURUN & MENINGKAT \\
\hline 2 & AGRO (PT BANK RAKYAT INDONESIA AGRONIAGA TbK) & MENINGKAT & MENURUN & MENURUN & MENURUN \\
\hline 3 & AGRS (PT BANK AGRIS Tbk) & MENINGKAT & MENINGKAT & MENINGKAT & MENURUN \\
\hline 4 & BABP (PT BANK MNC INTERNASIONAL TbK) & MENURUN & MENINGKAT & MENURUN & MENINGKAT \\
\hline 5 & BACA (PT BANK CAPITAL INDONESIA Tbk) & MENURUN & MENURUN & MENURUN & MENINGKAT \\
\hline 6 & BANK DANAMON & MENURUN & MENINGKAT & MENURUN & MENINGKAT \\
\hline 7 & BANK TJURST & MENINGKAT & MENURUN & MENURUN & MENINGKAT \\
\hline 8 & BBKP (BANK BUKOPIN) & MENINGKAT & MENURUN & MENURUN & MENINGKAT \\
\hline 9 & BBMD (PT BANK MESTIKA DHARMA TbK) & MENURUN & MENURUN & TETAP & MENINGKAT \\
\hline 10 & BBNI (BNI) & MENURUN & MENINGKAT & MENURUN & MENINGKAT \\
\hline 11 & BBRI (BRI) & MENINGKAT & MENINGKAT & TETAP & MENINGKAT \\
\hline 12 & BBTN ( PT BANK TABUNGAN NEGARA TbK) & MENINGKAT & MENURUN & MENURUN & MENINGKAT \\
\hline 13 & BEKS (BANK PEMBANGUNAN DAERAH BANTEN) & MENURUN & MENURUN & MENINGKAT & MENURUN \\
\hline 14 & BINA (BANK INA PERDANA) & MENINGKAT & MENURUN & MENINGKAT & MENURUN \\
\hline 15 & BJBR (BANK PEMBANGUNAN DAERAH JAWA BARAT) & MENURUN & MENURUN & MENINGKAT & MENINGKAT \\
\hline 16 & BJTM (BANK DAERAH JAWA TIMUR) & MENINGKAT & MENINGKAT & MENINGKAT & MENURUN \\
\hline 17 & BKSW (BANK QNB INDONESIA) & MENINGKAT & MENURUN & MENURUN & MENINGKAT \\
\hline 18 & BMAS (BANK MASPION INDONESIA TbK PT) & MENINGKAT & MENINGKAT & MENURUN & MENURUN \\
\hline 19 & BMRI (BANK MANDIRI PERSERO Tbk PT) & MENINGKAT & MENURUN & MENINGKAT & MENINGKAT \\
\hline 20 & BNBA (BANK BUMI ARTA) & MENINGKAT & MENURUN & MENINGKAT & MENINGKAT \\
\hline 21 & BNGA (BANK CIMB NIAGA) & MENURUN & MENINGKAT & MENINGKAT & MENINGKAT \\
\hline 22 & BNII (BANK MAYBANK INDONESIA TbK PT) & MENURUN & MENINGKAT & MENINGKAT & MENINGKAT \\
\hline 23 & BNLI (BANK PERMATA) & MENURUN & MENURUN & MENINGKAT & MENINGKAT \\
\hline 24 & BRIS (Bank BRIsyariah Tbk) & MENURUN & MENURUN & MENINGKAT & MENURUN \\
\hline 25 & BSIM (BANK SINARMAS) & MENURUN & MENURUN & MENINGKAT & MENINGKAT \\
\hline 26 & BTPN (Bank BTPN) & MENINGKAT & MENURUN & MENINGKAT & MENINGKAT \\
\hline 27 & BVIC (Bank Victoria International) & MENURUN & TETAP & MENURUN & MENINGKAT \\
\hline 28 & DNAR (BANK DINAR INDONESIA TbK) & MENURUN & MENINGKAT & MENURUN & MENURUN \\
\hline 29 & INPC (BANK ARTHA GRAHA INTERNASIONAL Tbk PT) & MENURUN & MENINGKAT & MENURUN & MENURUN \\
\hline 30 & MAYA (BANK MAYAPADA INTERNASIONAL Tbk) & MENINGKAT & MENINGKAT & MENURUN & MENINGKAT \\
\hline 31 & MCOR ( BANK CHINA CONSTRACTION INDONESIA ) & MENINGKAT & MENINGKAT & MENURUN & MENINGKAT \\
\hline 32 & MEGA ( BANK MEGA ) & MENINGKAT & MENURUN & MENINGKAT & MENINGKAT \\
\hline 33 & NISP( BANK OCBC) & MENINGKAT & MENURUN & MENINGKAT & MENURUN \\
\hline 34 & NOBU ( BANK NATIONAL NOBU) & MENINGKAT & MENURUN & MENURUN & MENINGKAT \\
\hline 35 & PNBN ( BANK PAN INDONESIA) & MENINGKAT & MENURUN & MENINGKAT & MENINGKAT \\
\hline 36 & PNBS ( BANK PANIN SYARIAH) & MENURUN & MENURUN & TETAP & TETAP \\
\hline 37 & SDRA ( BANK WOORI SAUDARA) & MENINGKAT & MENINGKAT & MENINGKAT & MENINGKAT \\
\hline & LDR MENINGKAT & 20 & 14 & 17 & 26 \\
\hline & LDR MENURUN & 17 & 22 & 17 & 10 \\
\hline & LDR TETAP & 0 & 1 & 3 & 1 \\
\hline & TOTAL & 37 & 37 & 37 & 37 \\
\hline & \% LDR MENINGKAT & $54.05 \%$ & $37.84 \%$ & $45.95 \%$ & $70.27 \%$ \\
\hline & \% LDR MENURUN & $45.95 \%$ & $59.46 \%$ & $45.95 \%$ & $27.03 \%$ \\
\hline & \% LDR TETAP & $0.00 \%$ & $2.70 \%$ & $8.11 \%$ & $2.70 \%$ \\
\hline & \% TOTAL & $100 \%$ & $100 \%$ & $100 \%$ & $100 \%$ \\
\hline
\end{tabular}

This ratio shows the ratio between the total loan with the total deposit of a bank. This ratio shows the level of liquidity of a bank. LDR that is too high indicates a decreased ability of liquidity. The summary of data processing results shows that the highest increase in LDR occurred in 2018, namely 26 banks or $70.27 \%$ of the total banks studied. The lowest increase occurred in 2016 , only 14 banks or $37.84 \%$ of the total banks studied. 


\section{Net Loans to Total Asset}

Table 3. Net Loans to Total Asset

\begin{tabular}{|c|c|c|c|c|c|}
\hline & KETERANGAN & 2015 & 2016 & 2017 & 2018 \\
\hline 1 & BSWD (BANK OF INDIA INDONESIA) & MENURUN & MENURUN & MENURUN & MENINGKAT \\
\hline 2 & AGRO (PT BANK RAKYAT INDONESIA AGRONIAGA Tbk ) & MENURUN & MENURUN & MENURUN & MENINGKAT \\
\hline 3 & AGRS (PT BANK AGRIS Tbk) & MENINGKAT & MENINGKAT & MENURUN & MENURUN \\
\hline 4 & BABP (PT BANK MNC INTERNASIONAL TbK) & MENURUN & MENINGKAT & MENINGKAT & MENINGKAT \\
\hline 5 & BACA (PT BANK CAPITAL INDONESIA TbK) & MENURUN & MENURUN & MENURUN & MENINGKAT \\
\hline 6 & BANK DANAMON & MENURUN & MENURUN & MENURUN & MENINGKAT \\
\hline 7 & BANK TJURST & MENINGKAT & MENURUN & MENURUN & MENURUN \\
\hline 8 & BBKP (BANK BUKOPIN) & MENINGKAT & MENURUN & MENURUN & MENINGKAT \\
\hline 9 & BBMD (PT BANK MESTIKA DHARMA Tbk) & TETAP & MENURUN & MENURUN & MENINGKAT \\
\hline 10 & BBNI (BNI) & MENURUN & MENINGKAT & MENURUN & MENINGKAT \\
\hline 11 & BBRI (BRI) & MENINGKAT & MENINGKAT & MENURUN & MENURUN \\
\hline 12 & BBTN ( PT BANK TABUNGAN NEGARA Tbk) & MENINGKAT & MENURUN & MENURUN & MENINGKAT \\
\hline 13 & BEKS (BANK PEMBANGUNAN DAERAH BANTEN) & MENURUN & MENURUN & MENINGKAT & MENURUN \\
\hline 14 & BINA (BANK INA PERDANA) & MENINGKAT & MENURUN & MENURUN & MENURUN \\
\hline 15 & BJBR (BANK PEMBANGUNAN DAERAH JAWA BARAT) & MENURUN & TETAP & TETAP & MENINGKAT \\
\hline 16 & BJTM (BANK DAERAH JAWA TIMUR) & MENURUN & MENURUN & MENURUN & MENURUN \\
\hline 17 & BKSW (BANK QNB INDONESIA) & MENINGKAT & MENURUN & MENURUN & MENURUN \\
\hline 18 & BMAS (BANK MASPION INDONESIA Tbk PT) & MENINGKAT & MENINGKAT & MENURUN & MENURUN \\
\hline 19 & BMRI (BANK MANDIRI PERSERO TbK PT) & MENINGKAT & MENURUN & MENINGKAT & MENINGKAT \\
\hline 20 & BNBA (BANK BUMI ARTA) & MENURUN & MENURUN & MENINGKAT & MENINGKAT \\
\hline 21 & BNGA (BANK CIMB NIAGA) & MENURUN & MENINGKAT & MENURUN & MENINGKAT \\
\hline 22 & BNII (BANK MAYBANK INDONESIA TbK PT) & MENURUN & MENURUN & MENURUN & MENINGKAT \\
\hline 23 & BNLI (BANK PERMATA) & MENURUN & MENURUN & MENURUN & MENINGKAT \\
\hline 24 & BRIS (Bank BRIsyariah Tbk) & MENURUN & MENURUN & MENINGKAT & MENURUN \\
\hline 25 & BSIM (BANK SINARMAS) & MENURUN & MENURUN & MENURUN & MENINGKAT \\
\hline 26 & BTPN (Bank BTPN) & MENINGKAT & MENURUN & MENINGKAT & MENURUN \\
\hline 27 & BVIC (Bank Victoria International) & MENURUN & MENURUN & MENURUN & MENURUN \\
\hline 28 & DNAR (BANK DINAR INDONESIA TbK) & MENINGKAT & MENINGKAT & MENURUN & MENURUN \\
\hline 29 & INPC (BANK ARTHA GRAHA INTERNASIONAL Tbk PT) & MENURUN & MENURUN & MENURUN & MENURUN \\
\hline 30 & MAYA (BANK MAYAPADA INTERNASIONAL TbK) & MENINGKAT & MENINGKAT & MENURUN & MENURUN \\
\hline 31 & MCOR ( BANK CHINA CONSTRACTION INDONESIA ) & MENINGKAT & MENURUN & MENURUN & MENINGKAT \\
\hline 32 & MEGA ( BANK MEGA) & MENURUN & MENURUN & MENINGKAT & MENINGKAT \\
\hline 33 & NISP( BANK OCBC) & MENINGKAT & MENURUN & MENINGKAT & MENURUN \\
\hline 34 & NOBU ( BANK NATIONAL NOBU) & MENINGKAT & MENURUN & MENINGKAT & MENINGKAT \\
\hline 35 & PNBN ( BANK PAN INDONESIA ) & MENURUN & MENURUN & MENURUN & MENINGKAT \\
\hline 36 & PNBS ( BANK PANIN SYARIAH) & MENURUN & TETAP & TETAP & TETAP \\
\hline 37 & SDRA ( BANK WOORI SAUDARA ) & MENINGKAT & MENINGKAT & MENINGKAT & MENURUN \\
\hline & NLTA MENINGKAT & 16 & 9 & 10 & 20 \\
\hline & NLTA MENURUN & 20 & 26 & 25 & 16 \\
\hline & NLTA TETAP & 1 & 2 & 2 & 1 \\
\hline & TOTAL & 37 & 37 & 37 & 37 \\
\hline & $\%$ NLTA MENINGKAT & $43.24 \%$ & $24.32 \%$ & $27.03 \%$ & $54.05 \%$ \\
\hline & $\%$ NLTA MENURUN & $54.05 \%$ & $70.27 \%$ & $67.57 \%$ & $43.24 \%$ \\
\hline & $\%$ NLTA TETAP & $2.70 \%$ & $5.41 \%$ & $5.41 \%$ & $2.70 \%$ \\
\hline & $\%$ TOTAL & $100 \%$ & $100 \%$ & $100 \%$ & $100 \%$ \\
\hline
\end{tabular}

Net Loan to Total Asset Ratio is a ratio used to measure the amount of loans disbursed with the amount of assets owned by a bank. The higher this ratio, shows the lower level of bank liquidity. The summary of data processing results shows that the highest increase in NLTA occurred in 2018, namely 20 banks or $54.05 \%$ of the total banks studied. The lowest increase occurred in 2016 with only 9 banks or $24.32 \%$ of the total banks studied. 


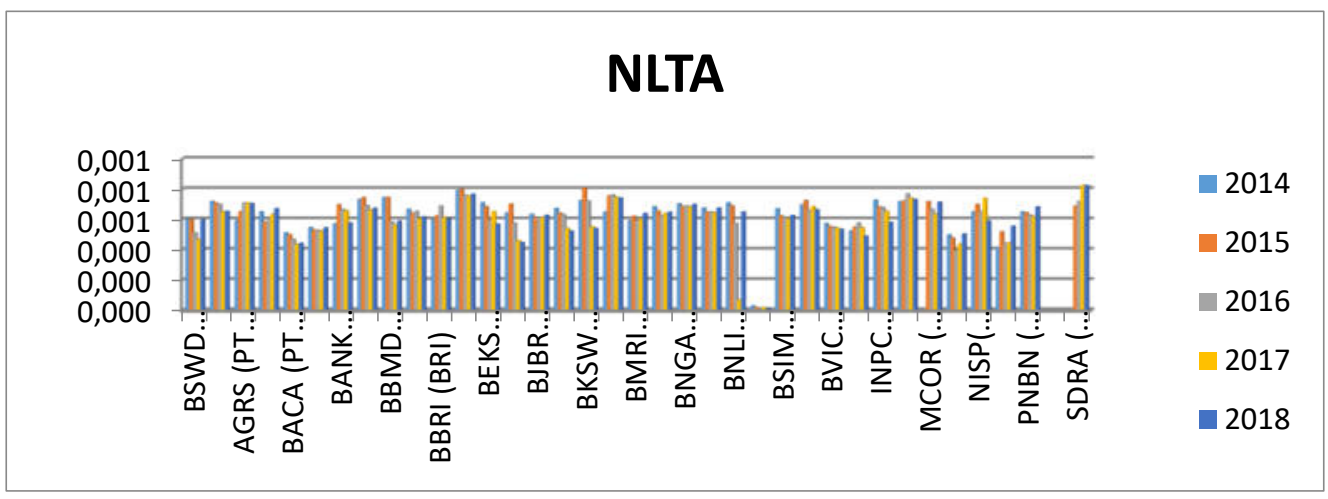

Figure 2. Net Loans to Total Asset

From the bar chart above it can be seen that there are no NLTA fluctuations that are too prominent.

\section{Non Performing Loans to Total Loans}

Table 3. Non Performing Loans to Total Loans

\begin{tabular}{|c|c|c|c|c|c|}
\hline & KETERANGAN & 2015 & 2016 & 2017 & 2018 \\
\hline 1 & BSWD (BANK OF INDIA INDONESIA) & MENINGKAT & MENURUN & MENURUN & MENURUN \\
\hline 2 & AGRO (PT BANK RAKYAT INDONESIA AGRONIAGA Tbk) & TETAP & MENINGKAT & MENURUN & MENINGKAT \\
\hline 3 & AGRS (PT BANK AGRIS Tbk) & MENINGKAT & MENINGKAT & MENINGKAT & MENURUN \\
\hline 4 & BABP (PT BANK MNC INTERNASIONAL TbK) & MENURUN & MENURUN & MENINGKAT & MENINGKAT \\
\hline 5 & BACA (PT BANK CAPITAL INDONESIA TbK) & MENINGKAT & MENINGKAT & MENURUN & MENINGKAT \\
\hline 6 & BANK DANAMON & MENINGKAT & MENURUN & MENINGKAT & MENINGKAT \\
\hline 7 & BANK TJURST & MENURUN & MENINGKAT & MENURUN & MENINGKAT \\
\hline 8 & BBKP (BANK BUKOPIN) & MENURUN & MENINGKAT & MENINGKAT & MENURUN \\
\hline 9 & BBMD (PT BANK MESTIKA DHARMA Tbk) & MENURUN & MENINGKAT & MENURUN & MENURUN \\
\hline 10 & BBNI (BNI) & MENINGKAT & MENURUN & MENINGKAT & MENINGKAT \\
\hline 11 & BBRI (BRI) & MENINGKAT & MENINGKAT & MENINGKAT & MENINGKAT \\
\hline 12 & BBTN ( PT BANK TABUNGAN NEGARA Tbk) & MENURUN & MENURUN & MENURUN & MENURUN \\
\hline 13 & BEKS (BANK PEMBANGUNAN DAERAH BANTEN) & MENINGKAT & MENURUN & MENURUN & MENINGKAT \\
\hline 14 & BINA (BANK INA PERDANA) & MENURUN & MENINGKAT & MENURUN & MENURUN \\
\hline 15 & BJBR (BANK PEMBANGUNAN DAERAH JAWA BARAT) & MENURUN & MENURUN & MENINGKAT & MENURUN \\
\hline 16 & BJTM (BANK DAERAH JAWA TIMUR) & MENINGKAT & MENURUN & MENURUN & MENINGKAT \\
\hline 17 & BKSW (BANK QNB INDONESIA) & MENINGKAT & MENINGKAT & MENINGKAT & MENINGKAT \\
\hline 18 & BMAS (BANK MASPION INDONESIA TbK PT) & MENURUN & MENINGKAT & MENINGKAT & MENINGKAT \\
\hline 19 & BMRI (BANK MANDIRI PERSERO TbK PT) & MENINGKAT & MENINGKAT & MENURUN & MENINGKAT \\
\hline 20 & BNBA (BANK BUMI ARTA) & MENURUN & MENURUN & MENINGKAT & MENURUN \\
\hline 21 & BNGA (BANK CIMB NIAGA) & MENINGKAT & MENURUN & MENINGKAT & MENURUN \\
\hline 22 & BNII (BANK MAYBANK INDONESIA TbK PT) & MENINGKAT & MENURUN & MENURUN & MENURUN \\
\hline 23 & BNLI (BANK PERMATA) & MENINGKAT & MENINGKAT & MENURUN & MENINGKAT \\
\hline 24 & BRIS (Bank BRIsyariah Tbk) & MENURUN & MENURUN & MENINGKAT & MENURUN \\
\hline 25 & BSIM (BANK SINARMAS) & MENINGKAT & MENURUN & MENINGKAT & MENURUN \\
\hline 26 & BTPN (Bank BTPN) & MENURUN & MENURUN & MENINGKAT & MENINGKAT \\
\hline 27 & BVIC (Bank Victoria International) & MENINGKAT & MENURUN & MENURUN & MENURUN \\
\hline 28 & DNAR (BANK DINAR INDONESIA Tbk) & MENURUN & MENINGKAT & MENINGKAT & MENURUN \\
\hline 29 & INPC (BANK ARTHA GRAHA INTERNASIONAL TbK PT) & MENURUN & MENINGKAT & MENINGKAT & MENURUN \\
\hline 30 & MAYA (BANK MAYAPADA INTERNASIONAL TbK) & MENINGKAT & MENURUN & MENINGKAT & MENURUN \\
\hline 31 & MCOR ( BANK CHINA CONSTRACTION INDONESIA ) & MENURUN & MENINGKAT & MENURUN & MENURUN \\
\hline 32 & MEGA ( BANK MEGA) & MENINGKAT & MENINGKAT & MENURUN & MENURUN \\
\hline 33 & NISP(BANK OCBC) & MENURUN & MENURUN & MENURUN & MENINGKAT \\
\hline 34 & NOBU ( BANK NATIONAL NOBU) & TETAP & TETAP & MENINGKAT & MENINGKAT \\
\hline 35 & PNBN ( BANK PAN INDONESIA) & MENURUN & MENINGKAT & MENURUN & MENINGKAT \\
\hline 36 & PNBS ( BANK PANIN SYARIAH) & TETAP & TETAP & TETAP & TETAP \\
\hline 37 & SDRA ( BANK WOORI SAUDARA ) & MENURUN & MENURUN & MENURUN & MENINGKAT \\
\hline & NPLTL MENINGKAT & 17 & 17 & 18 & 18 \\
\hline & NPLTL MENURUN & 17 & 18 & 18 & 18 \\
\hline & NPLTL TETAP & 3 & 2 & 1 & 1 \\
\hline & TOTAL & 37 & 37 & 37 & 37 \\
\hline & $\%$ NPLTL MENINGKAT & $45.95 \%$ & $45.95 \%$ & $48.65 \%$ & $48.65 \%$ \\
\hline & $\%$ NPLTL MENURUN & $45.95 \%$ & $48.65 \%$ & $48.65 \%$ & $48.65 \%$ \\
\hline & $\%$ NPLTL TETAP & $8.11 \%$ & $5.41 \%$ & $2.70 \%$ & $2.70 \%$ \\
\hline & \% TOTAL & $100 \%$ & $100 \%$ & $100 \%$ & $100 \%$ \\
\hline
\end{tabular}


This ratio shows the ratio between the number of bad loans and the total loans given by banks. From the table above it can be seen that the performance in 2017 and 2018 is the same, namely there are 18 banks or $48.65 \%$ of banks experiencing an increase in NPLTL. In 2015 and 2016 also had the same performance, namely 17 banks or $45.95 \%$ of banks that experienced an increase in NPLTL.

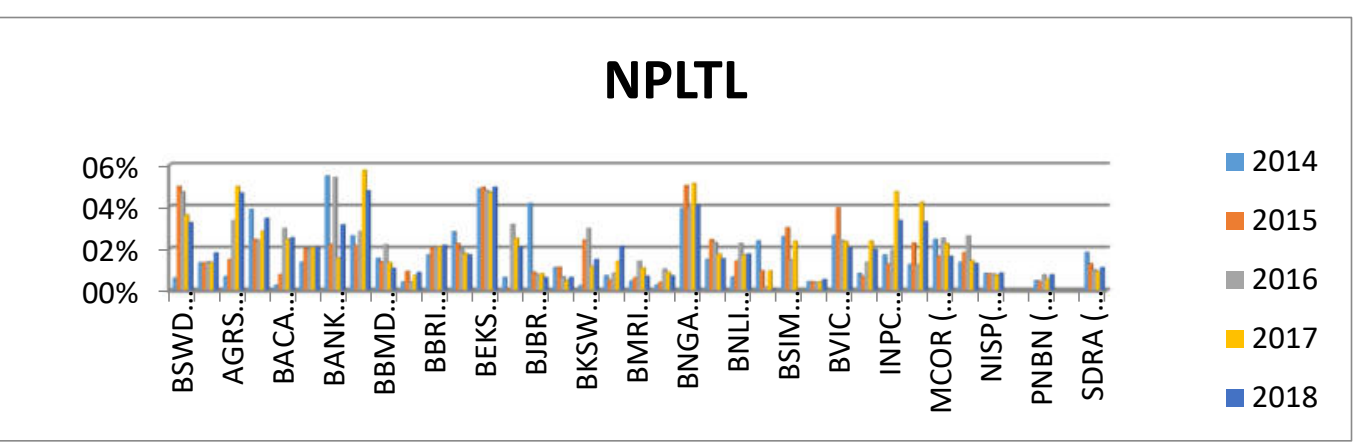

Figure 3. Non Performing Loans to Total Loans

From the bar chart above, it can be seen that NPLTL fluctuations are very volatile.

\section{Discussion}

\section{Return on Asset}

This ratio shows the rate of return (profit) compared to total assets. The higher this ratio shows the better performance. The summary of data processing results shows that the most decline in ROA occurred in 2017, namely 23 banks or $62.16 \%$ of the total banks studied. Interestingly, in 2018 the decline in ROA occurred only in 17 banks or $45.95 \%$ of the total banks studied, lower than in 2015 which reached 20 banks or $54.05 \%$ and in 2017 which became 23 banks or $62.16 \%$ of the total banks studied.

From the above data, it can be concluded that the decline in the value of the rupiah that occurred at the beginning of 2018 did not or did not have a worse impact in the year concerned, compared to previous years. It could be that the impact on ROA will be seen in the period after 2018 .

\section{Loan to Deposit Ratio}

This ratio shows the ratio between the total loan with the total deposit of a bank. This ratio shows the level of liquidity of a bank. LDR that is too high indicates a decreased ability of liquidity. The summary of data processing results shows that the highest increase in LDR occurred in 2018, namely 26 banks or $70.27 \%$ of the total banks studied. The lowest increase occurred in 2016, only 14 banks or $37.84 \%$ of the total banks studied.

From the above data it can be seen that bank liquidity has decreased the most in 2018, which indicates that the decline in the value of the rupiah in early 2018 has affected bank liquidity in the year concerned.

\section{Net Loans to Total Asset}

Net Loan to Total Asset Ratio is a ratio used to measure the amount of loans disbursed with the amount of assets owned by a bank. The higher this ratio, shows the lower level of bank liquidity. The summary of data processing results shows that the highest increase in NLTA occurred in 2018, namely 20 banks or $54.05 \%$ of the total banks studied. The lowest increase occurred in 2016 with only 9 banks or $24.32 \%$ of the total banks studied.

From the above data, it can be concluded that the decline in the value of the rupiah that occurred at the beginning of 2018 had affected bank liquidity in the period concerned.

\section{Non Performing Loans to Total Loans}

This ratio shows the ratio between the number of bad loans and the total loans given by banks. From the table above it can be seen that the performance in 2017 and 2018 is the same, namely there are 18 banks or $48.65 \%$ of 
banks experiencing an increase in NPLTL. In 2015 and 2016 also had the same performance, namely 17 banks or $45.95 \%$ of banks that experienced an increase in NPLTL.

From the above data it cannot be concluded whether the decline in the value of the rupiah in early 2018 affects the NPLTL or not.

\section{Conclusions and Suggestions}

From the discussion above, it can be concluded that the decline in the rupiah in early 2018 could immediately be seen as having an impact on bank liquidity in the year concerned. while the ROA is not or has not been seen, while for non-performing loans performance decreases have occurred from 2017.

Suggested that further studies increase the span of the observation period after 2018, to see whether ROA and nonperforming loans are really not affected by the decline in the value of the rupiah or not.

\section{References}

Aburime, T. U. 2008. Determinants of Bank Profitability: Macroeconomic Evidence from Nigeria.

Agustio, Alfado. 2018. Kondisi Perbankan di Tengah Pelemahan Rupiah, MasihkahAman?.https://www.cnbcindonesia.com/market/20180922131902-17-34324/_ kondisiperbankan-di-tengah-pelemahan-rupiah-masihkah-aman. Diakses tanggal 15 Agustus 2019 pukul

Ahmed, M., B. 2009. Measuring the Performance of Islamic Banks by Adapting Conventional Ratios. German University in Cairo Faculty of Management Technology Working Paper no. 16 pp 1-26.

Casu, B., Girardone C., Bank Competition, Concentration and Efficiency in the Single European Market. The Manchester School vol. 74 no. 4 pp. 441-468

Edward, I. 1968. Financial Ratios, Discriminant Analysis and the Prediction of Corporate Bankruptcy. Altman The Journal of Finance, nol. 23, no. 4. pp. 589-609.

Francis, M.E. 2013. Determinants of Commercial Bank Profitability in Sub-Saharan Africa. International Journal of Economics and Finance vol. 5 no. 9 pp. 134-147.

Gitman, L.J. 2004. Principles of Managerial Finance. 10th Edition. S. l, Pearson Education, Australia.

Islam, Md Aminul. 2014. An Analysis of the Financial Performance of National Bank Limited Using Financial Ratio. Journal of Behavioural Economics, Finance, Entrepreneurship, Accounting and Transport vol. 2. no.5 pp. 121-129.

Khan, MY and P K Jain. 2012. Financial Management. New Delhi. India : Mc. Graw-Hill.

Ohlson, James A.1980. Financial Ratios and the Probabilistic Prediction of Bankruptcy. Journal of Accounting Research vol. 18 no. 1 pp. 109-131

Ross, S.A., Westerfield, R.W., \& Jordan, B.D. 2000. Fundamentals Of Corporate Finance. New York : Mc GrawHill.

S. K. Misra and P. K. Aspal, 2013. A camel model analysis of state bank group. World Journal of Social Sciences, vol. 3, no.4, pp. 36-55.

Situmorang, Angggun P. 2018. Kata Sri Mulyani soal Peranan Perbankan dalam Mendorong Ekonomi RI.https://www.liputan6.com/bisnis/read/3526017/kata-sri-mulyani-soalperanan-perbankan-dalammendorong- 
ekonomiri?utmexpid=.9Z4i5ypGQeGiS7w9arwTvQ.0\&utm_referrer=https $\% 3 \mathrm{~A} \% 2 \mathrm{~F} \% 2 \mathrm{Fwww}$.google.co m\%2F. Diakses tanggal 15 Agustus 2019 pukul

Tekatel, W. L., 2019. Financial Performance Analysis (MBA project). Thesis Department of Applied Mathematics Andhra University

Thygerson, Kenneth J. 1995. Management of financial institutions. New York: HarperCollins College Publishers. Van Horne, J, \& John Wachowicz, J. 2009. Financial Management Prinsip-Prinsip Manajemen Keuangan Edisi 13, Penerjemah Quratulain Mubarakah, Jakarta : Salemba Empat.

Zerayehu SE, Kagnew WT, Teshome KA (2013). Competition in Ethiopian banking industry. African Journal of Economics 1(5):176-190. 